\title{
Analysis of the neutralino system in supersymmetric theories
}

\author{
S.Y. Choi ${ }^{1}$, J. Kalinowski ${ }^{2}$, G. Moortgat-Pick ${ }^{3}$, P.M. Zerwas ${ }^{3}$ \\ 1 Department of Physics, Chonbuk National University, Chonju 561-756, Korea \\ 2 Instytut Fizyki Teoretycznej, Uniwersytet Warszawski, 00681 Warsaw, Poland \\ 3 Deutsches Elektronen-Synchrotron DESY, 22603 Hamburg, Germany
}

Eur. Phys. J. C 22, 563-579 (2001) - DOI 10.1007/s100520100808

Published online: 21 November 2001 /

Addendum published online: 5 April 2002 - (c) Springer-Verlag / Società Italiana di Fisica 2002

\begin{abstract}
In the preceding reference [1] we have shown how the fundamental gaugino and higgsino parameters of the chargino and neutralino system in supersymmetric theories can be determined in high-precision experiments at $e^{+} e^{-}$linear colliders. Within the Minimal Supersymmetric Standard Model these parameters can be reconstructed completely even if only the light charginos $\tilde{\chi}_{1}^{ \pm}$and the light neutralinos $\tilde{\chi}_{1}^{0}$ and $\tilde{\chi}_{2}^{0}$ are kinematically accessible in the initial phase of these machines, as demonstrated in this Addendum.
\end{abstract}

\section{The basis}

The fundamental parameters of the gaugino/higgsino sector in supersymmetric theories, the $\mathrm{U}(1)$ and $\mathrm{SU}(2)$ gaugino masses $M_{1}$ and $M_{2}$, and the higgsino mass $\mu$, can be determined very accurately in experiments at prospective $e^{+} e^{-}$linear colliders. This has been demonstrated in the elaborate analysis of Ref. [1]. In the initial phase of the colliders, a total energy of $\sqrt{s}=500 \mathrm{GeV}$, raised later to $\sim 1 \mathrm{TeV}$, is planned to be reached with a high integrated luminosity of $\sim 1 \mathrm{ab}^{-1}$ within a few years [2]; the electron and positron beams are planned to be polarized with a degree of 80 and $60 \%$, respectively [3].

In many scenarios it is expected [4] that the light charginos $\tilde{\chi}_{1}^{ \pm}$and the two lightest neutralinos $\tilde{\chi}_{1}^{0}$ and $\tilde{\chi}_{2}^{0}$ can be accessed kinematically ${ }^{1}$ in the initial phase of the colliders. In this Addendum to [1] it will be shown that in the Minimal Supersymmetric Standard Model (MSSM) the gaugino and higgsino mass parameters can be reconstructed completely in this case ${ }^{2}$, even in $\mathrm{CP}$ noninvariant versions of the model, by measuring the properties of the light particle set $\left\{\tilde{\chi}_{1}^{ \pm} ; \tilde{\chi}_{1}^{0}, \tilde{\chi}_{2}^{0}\right\}$.

In $e^{+} e^{-}$collisions, charginos and neutralinos can be produced in diagonal and mixed pairs among which the reactions, giving rise to visible final states,

$$
e^{+} e^{-} \rightarrow \tilde{\chi}_{1}^{+} \tilde{\chi}_{1}^{-}
$$

\footnotetext{
1 In most supergravity inspired scenarios, for example, mass relations of the type $m_{\tilde{\chi}_{1}^{ \pm}} \sim m_{\tilde{\chi}_{2}^{0}} \sim 2 m_{\tilde{\chi}_{1}^{0}}$ are realized in the chargino/neutralino sector

2 For different strategies of determining the fundamental pa-
} rameters see [5] and references therein

$$
e^{+} e^{-} \rightarrow \tilde{\chi}_{1}^{0} \tilde{\chi}_{2}^{0}
$$

are of particular experimental interest in the present context.

In standard definition $[1,6,7]$, the diagonalization of the chargino matrix in the MSSM

$$
\mathcal{M}_{C}=\left(\begin{array}{cc}
M_{2} & \sqrt{2} m_{W} c_{\beta} \\
\sqrt{2} m_{W} s_{\beta} & \mu
\end{array}\right)
$$

generates the light and heavy states $\tilde{\chi}_{i}^{ \pm}(i=1,2)$, while diagonalizing the neutralino mass matrix

$$
\mathcal{M}_{N}=\left(\begin{array}{cccc}
M_{1} & 0 & -m_{Z} c_{\beta} s_{W} & m_{Z} s_{\beta} s_{W} \\
0 & M_{2} & m_{Z} c_{\beta} c_{W} & -m_{Z} s_{\beta} c_{W} \\
-m_{Z} c_{\beta} s_{W} & m_{Z} c_{\beta} c_{W} & 0 & -\mu \\
m_{Z} s_{\beta} s_{W} & -m_{Z} s_{\beta} c_{W} & -\mu & 0
\end{array}\right)
$$

leads to four neutralino states $\tilde{\chi}_{i}^{0}(i=1,2,3,4)$, ordered sequentially with rising mass. The coefficients $s_{\beta}=\sin \beta$, $c_{\beta}=\cos \beta$ are given by the ratio of the vacuum expectation values of the Higgs fields, $\tan \beta=v_{2} / v_{1}$, and $s_{W}, c_{W}$ are the sine and cosine of the electroweak mixing angle. In $\mathrm{CP}-$ noninvariant theories, the mass parameters are complex. By reparametrization of the field basis, the $\mathrm{SU}(2)$ mass parameter $M_{2}$ can be set real and positive, while the $\mathrm{U}(1)$ mass parameter $M_{1}$ is assigned the phase $\Phi_{1}$, and the higgsino mass parameter $\mu$ the phase $\Phi_{\mu}$.

The examples presented later, will be based on a single reference point for a $\mathrm{CP}$ non-invariant extension of the 
MSSM, compatible with all experimental constraints $[8$, 9],

$$
\begin{aligned}
\mathrm{RP} & :\left(\left|M_{1}\right|, M_{2},|\mu| ; \Phi_{1}, \Phi_{\mu} ; \tan \beta\right) \\
& =\left(100.5 \mathrm{GeV}, 190.8 \mathrm{GeV}, 365.1 \mathrm{GeV} ; \frac{\pi}{3}, \frac{\pi}{8} ; 10\right)
\end{aligned}
$$

These fundamental parameters generate the following light chargino and neutralino masses,

$$
\begin{aligned}
m_{\tilde{\chi}_{1}^{ \pm}} & =176.0 \mathrm{GeV} \\
m_{\tilde{\chi}_{1}^{0}} & =98.7 \mathrm{GeV} \\
m_{\tilde{\chi}_{2}^{0}} & =176.3 \mathrm{GeV}
\end{aligned}
$$

while the heavy masses are given by

$$
\begin{aligned}
m_{\tilde{\chi}_{2}^{ \pm}} & =389.3 \mathrm{GeV} \\
m_{\tilde{\chi}_{3}^{0}} & =371.8 \mathrm{GeV} \\
m_{\tilde{\chi}_{4}^{0}} & =388.2 \mathrm{GeV}
\end{aligned}
$$

The cross sections depend on the sneutrino and selectron masses which we assume, for the sake of simplicity, to be measured in threshold scans :

$$
\begin{aligned}
& m_{\tilde{\nu}_{L}}=192.8 \mathrm{GeV} \\
& m_{\tilde{e}_{L}}=208.7 \mathrm{GeV} \\
& m_{\tilde{e}_{R}}=144.1 \mathrm{GeV}
\end{aligned}
$$

[Angular correlations in the production of chargino/ neutralino states can be exploited otherwise to determine the slepton masses [10].] The cross sections for chargino and neutralino pair-production with polarized beams are big at $\sqrt{s}=500 \mathrm{GeV}$,

$$
\begin{array}{llrl}
\sigma_{L}\left\{\tilde{\chi}_{1}^{+} \tilde{\chi}_{1}^{-}\right\} & =679.5 \mathrm{fb} & & \sigma_{R}\left\{\tilde{\chi}_{1}^{+} \tilde{\chi}_{1}^{-}\right\}=1.04 \mathrm{fb} \\
\sigma_{L}\left\{\tilde{\chi}_{1}^{0} \tilde{\chi}_{2}^{0}\right\}=327.9 \mathrm{fb} & \sigma_{R}\left\{\tilde{\chi}_{1}^{0} \tilde{\chi}_{2}^{0}\right\}=16.4 \mathrm{fb}
\end{array}
$$

so that sufficiently large ensembles of events, between $\sim 7 \times 10^{5}$ and $1 \times 10^{3}$ events for $\tilde{\chi}_{1}^{+} \tilde{\chi}_{1}^{-}$and $\tilde{\chi}_{1}^{0} \tilde{\chi}_{2}^{0}$, will be generated $^{3}$, allowing the analysis of the properties of the chargino $\tilde{\chi}_{1}^{ \pm}$and the neutralinos $\tilde{\chi}_{1,2}^{0}$ at great detail.

\section{The chargino system}

Defining the mixing angles in the unitary matrices diagonalizing the chargino mass matrix $\mathcal{M}_{C}$ by $\phi_{L}$ and $\phi_{R}$ for the left- and right-chiral fields, the fundamental SUSY parameters $M_{2},|\mu|, \cos \Phi_{\mu}$ and $\tan \beta$ can be derived from the chargino masses and the cosines $c_{2 L, R}=\cos 2 \phi_{L, R}$ of the mixing angles,

$$
M_{2}=m_{W} \sqrt{\Sigma-\Delta\left(c_{2 L}+c_{2 R}\right)}
$$

${ }^{3}$ Information derived from other open channels like $\tilde{\chi}_{2}^{0} \tilde{\chi}_{2}^{0}$, etc, can be used to refine the analysis

$$
\begin{aligned}
|\mu| & =m_{W} \sqrt{\Sigma+\Delta\left(c_{2 L}+c_{2 R}\right)} \\
\cos \Phi_{\mu} & =\frac{\Delta^{2}\left(2-c_{2 L}^{2}-c_{2 R}^{2}\right)-\Sigma}{\sqrt{\left[1-\Delta^{2}\left(c_{2 L}-c_{2 R}\right)^{2}\right]\left[\Sigma^{2}-\Delta^{2}\left(c_{2 L}+c_{2 R}\right)^{2}\right]}} \\
\tan \beta & =\sqrt{\frac{1-\Delta\left(c_{2 L}-c_{2 R}\right)}{1+\Delta\left(c_{2 L}-c_{2 R}\right)}}
\end{aligned}
$$

where the dimensionless quantities

$$
\begin{aligned}
& \Sigma=\left[m_{\tilde{\chi}_{2}^{ \pm}}^{2}+m_{\tilde{\chi}_{1}^{ \pm}}^{2}-2 m_{W}^{2}\right] / 2 m_{W}^{2} \quad \text { and } \\
& \Delta=\left[m_{\tilde{\chi}_{2}^{ \pm}}^{2}-m_{\tilde{\chi}_{1}^{ \pm}}^{2}\right] / 4 m_{W}^{2}
\end{aligned}
$$

have been introduced for notational convenience.

If only the light charginos $\tilde{\chi}_{1}^{ \pm}$can be produced, besides the mass $m_{\tilde{\chi}_{1}^{ \pm}}$, both the mixing parameters $\cos 2 \phi_{L, R}$ can be measured nevertheless $[6,7]$. The $\cos 2 \phi_{L, R}$ can be determined uniquely if the polarized cross sections are measured at one energy including transverse beam polarization, or else if the longitudinally polarized cross sections are measured at two different energies.

It is apparent from (14) that the heavy chargino mass is bounded from above after $m_{\tilde{\chi}_{1}^{ \pm}}$and $\cos 2 \phi_{L, R}$ are measured experimentally. At the same time, it is bounded from below by not observing the heavy chargino in mixed light-heavy pair production. The ensuing constraint on the heavy chargino mass

$$
\begin{aligned}
\frac{1}{2} \sqrt{s}-m_{\tilde{\chi}_{1}^{ \pm}} & \leq m_{\tilde{\chi}_{2}^{ \pm}} \\
& \leq \sqrt{m_{\tilde{\chi}_{1}^{ \pm}}^{2}+4 m_{W}^{2} /\left|\cos 2 \phi_{L}-\cos 2 \phi_{R}\right|}
\end{aligned}
$$

is quite restrictive; the upper bound can still be improved by exploiting the slightly more restrictive, but algebraically more complicated bound derived from $\left|\cos \Phi_{\mu}\right| \leq$ 1 in (13). For the example introduced above, a narrow window of $324.0 \mathrm{GeV} \leq m_{\tilde{\chi}_{2}^{ \pm}} \leq 389.7 \mathrm{GeV}$ is predicted after initial experimentation at the energy $\sqrt{s}=500 \mathrm{GeV}$.

If both the light chargino mass $m_{\tilde{\chi}_{1}^{ \pm}}$and the heavy chargino mass $m_{\tilde{\chi}_{2}^{ \pm}}$can be measured, the fundamental parameters $\left\{M_{2}, \mu ; \tan \beta\right\}$ can be extracted unambiguously. However, if $\tilde{\chi}_{2}^{ \pm}$is not accessible, it depends on the CP properties of the higgsino sector whether they can be determined or not in the chargino system alone.

(A) If the higgsino sector is $C P$ invariant $^{4}$, (13) can be exploited to determine $m_{\tilde{\chi}_{2}^{ \pm}}^{2}$ from $\cos \Phi_{\mu}= \pm 1$, up to at most a two-fold ambiguity [6,7]. This ambiguity can be resolved if other observables can be evaluated, notabene the mixed-pair $\tilde{\chi}_{1}^{0} \tilde{\chi}_{2}^{0}$ production cross sections.

(B) If $\tilde{\chi}_{2}^{ \pm}$is not accessible, the parameters in (11-14) cannot be determined in a $C P$ non-invariant theory in

\footnotetext{
4 Analyses of electric dipole moments strongly suggest that
} $\mathrm{CP}$ violation in the higgsino sector will be very small in the MSSM if this sector is non-invariant at all $[8,9]$ 
the chargino sector alone. They remain dependent on the unknown heavy chargino mass $m_{\tilde{\chi}_{2}^{ \pm}}$. Two trajectories are generated in $\left\{M_{2}, \mu ; \tan \beta\right\}$ space, parametrized by $m_{\tilde{\chi}_{2}^{ \pm}}$and classified by the two values $\Phi_{\mu}$ and $\left(2 \pi-\Phi_{\mu}\right)$ for the phase of the higgsino mass parameter, i.e. the sign of $\sin \Phi_{\mu}$. It will be shown in the next section that the analysis of the two light neutralino states $\tilde{\chi}_{1}^{0}$ and $\tilde{\chi}_{2}^{0}$ can be used to predict the heavy chargino mass $m_{\tilde{\chi}_{2}^{ \pm}}$in the MSSM. The phase ambiguity can be resolved ${ }^{5}$ by measuring the sign of $\mathrm{CP}$-odd observables associated with normal $\tilde{\chi}_{2}^{0}$ polarization in $\tilde{\chi}_{1}^{0} \tilde{\chi}_{2}^{0}$ pair production [9]. Subsequently the entire set of fundamental gaugino and higgsino parameters can be determined uniquely.

\section{The neutralino system}

The symmetric neutralino mass matrix $\mathcal{M}_{N}$ is diagonalized by a unitary matrix, defined such that the mass eigenvalues $m_{\tilde{\chi}_{i}^{0}}$ of the four Majorana fields $\tilde{\chi}_{i}^{0}$ are positive.

The squared mass eigenvalues of $\mathcal{M}_{N} \mathcal{M}_{N}^{\dagger}$ are solutions of the characteristic equations [1]

$m_{\tilde{\chi}_{i}^{0}}^{8}-a m_{\tilde{\chi}_{i}^{0}}^{6}+b m_{\tilde{\chi}_{i}^{0}}^{4}-c m_{\tilde{\chi}_{i}^{0}}^{2}+d=0 \quad$ for $\quad i=1,2,3,4$

with the invariants $a, b, c$ and $d$ given by the fundamental $\mathrm{SU}(2)$ and $\mathrm{U}(1)$ gaugino mass parameters $M_{2}$ and $M_{1}$, and the higgsino mass parameter $\mu$, i.e. the moduli $M_{2},\left|M_{1}\right|$, $|\mu|$ and the phases $\Phi_{1}, \Phi_{\mu}$. Each of the four invariants $a, b, c$ and $d$ is a binomial of $\Re \mathrm{e} M_{1}=\left|M_{1}\right| \cos \Phi_{1}$ and ऽm $M_{1}=\left|M_{1}\right| \sin \Phi_{1}$. Therefore, each of the characteristic equations in the set (17) for the neutralino mass squared $m_{\tilde{\chi}_{i}^{0}}^{2}$ can be rewritten in the form

$$
\begin{aligned}
& \left(\Re \mathrm{e} M_{1}\right)^{2}+\left(\Im \mathrm{m} M_{1}\right)^{2}+u_{i} \Re \mathrm{e} M_{1}+v_{i} \Im \mathrm{m} M_{1}=w_{i} \\
& \text { for } \quad i=1,2,3,4
\end{aligned}
$$

The coefficients $u_{i}, v_{i}$ and $w_{i}$ are functions of the parameters $M_{2},|\mu|, \Phi_{\mu}, \tan \beta$ and the mass eigenvalue $m_{\tilde{\chi}_{i}^{0}}^{2}$ for fixed $i$. The coefficient $v_{i}$ is necessarily proportional to $\sin \Phi_{\mu}$ because physical neutralino masses are $\mathrm{CP}-$ even; the sign ambiguity for $\sin \Phi_{\mu}$, a result of the two-fold $\cos$ solution $\Phi_{\mu} \leftrightarrow\left(2 \pi-\Phi_{\mu}\right)$, transfers to the associated sign ambiguity in the $\mathrm{CP}$-odd quantity $\Im \mathrm{m} M_{1}$, i.e. in $\sin \Phi_{1}$.

\section{Reconstruction of the fundamental parameters}

The characteristic equation (18) defines a circle in the $\left\{\Re \mathrm{e} M_{1}, \Im \mathrm{m} M_{1}\right\}$ plane for each neutralino mass $m_{\tilde{\chi}_{i}^{0}}$. With only two light neutralino masses $m_{\tilde{\chi}_{1}^{0}}$ and $m_{\tilde{\chi}_{2}^{0}}$ measured, we are left with a two-fold ambiguity marked by the two, black and open, dots in Fig. 1.

5 If not resolved, the two-fold ambiguity will propagate to the final set $\left\{M_{1}, M_{2} ; \mu\right\}$ with the sign of $\Im m M_{1}$, coupled to the sign of $\Im \mathrm{m} \mu$, remaining undetermined, i.e. the $\operatorname{sign}$ of $\sin \Phi_{1}$ coupled to the sign of $\sin \Phi_{\mu}$

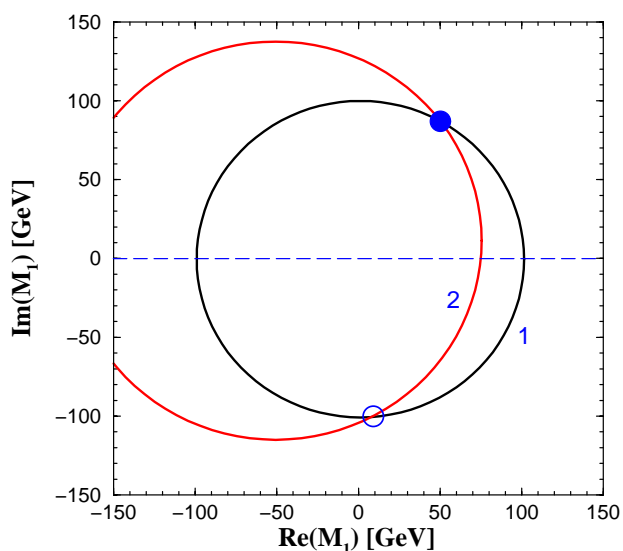

Fig. 1. The contours of two measured neutralino masses $m_{\tilde{\chi}_{1}^{0}}$ and $m_{\tilde{\chi}_{2}^{0}}$ in the $\left\{\Re \mathrm{e} M_{1}, \Im \mathrm{m} M_{1}\right\}$ plane; the parameter set $\left\{M_{2}=190.8 \mathrm{GeV} ;|\mu|=365.1 \mathrm{GeV}, \Phi_{\mu}=\pi / 8 ; \tan \beta=10\right\}$ is assumed to be known from the chargino sector. [The second possible solution of (18), with the circles reflected at the broken null-line, can be rejected by measuring the sign of $\sin \Phi_{\mu}$, related to the $\operatorname{sign}$ of $\sin \Phi_{1}$ or $\Im m M_{1}$.] (a) $\operatorname{Re}\left(\mathrm{M}_{1}\right)[\mathrm{GeV}]$

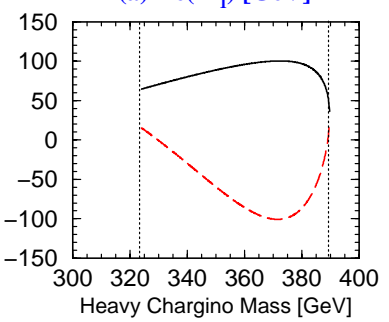

(c) $\sigma_{\mathrm{L}}\{12\}[\mathbf{f b}]$

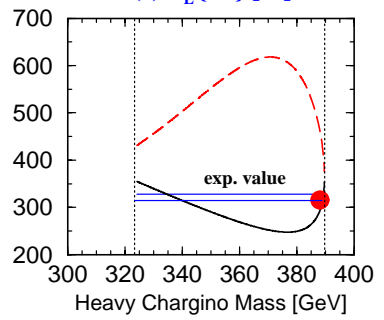

(b) $\operatorname{Im}\left(\mathrm{M}_{1}\right)[\mathrm{GeV}]$

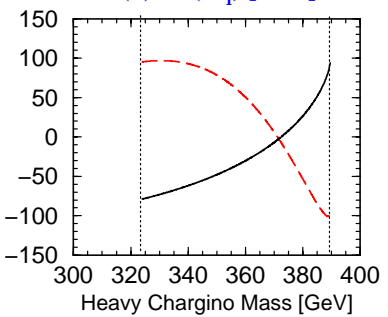

(d) $\sigma_{\mathrm{L}}$ expanded

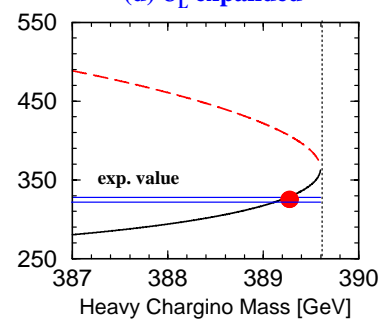

Fig. 2. a, b The two sets of $\left\{\Re \mathrm{e} M_{1}, \Im \mathrm{m} M_{1}\right\}$ denoting the crossing points of the two circles for the measured neutralino masses in Fig. 1 within the allowed window; c the corresponding cross sections $\sigma_{L}\left\{\tilde{\chi}_{1}^{0} \tilde{\chi}_{2}^{0}\right\}$ as functions of the heavy chargino mass for its entire allowed mass range, and $\mathbf{d}$ magnified for the unique solution $m_{\tilde{\chi}_{2}^{ \pm}}=389.3 \mathrm{GeV}$. [The other solution to $\sigma_{L}\left\{\tilde{\chi}_{1}^{0} \tilde{\chi}_{2}^{0}\right\}$ can be excluded by the measurement of $\sigma_{R}\left\{\tilde{\chi}_{1}^{0} \tilde{\chi}_{2}^{0}\right\}$.] The experimental cross section for $L$ polarization is denoted by the horizontal lines in the lower panels

(A) In the $C P$ invariant version of the MSSM, the measurements of $m_{\tilde{\chi}_{1}^{ \pm}}$and $\cos 2 \phi_{L, R}$ lead to at most a two-fold ambiguity in $\left\{M_{2}, \mu ; \tan \beta\right\}$. Inserting the two neutralino masses $m_{\tilde{\chi}_{1,2}^{0}}$ in the set (18), adjusted properly by setting $\Im \mathrm{m} M_{1}=0$, this induces at most a two-fold sign ambiguity in $M_{1}$, real in CP-invariant theories. This ambiguity can finally be resolved by measuring the cross sections for $\tilde{\chi}_{1}^{0} \tilde{\chi}_{2}^{0}$ pair production. 


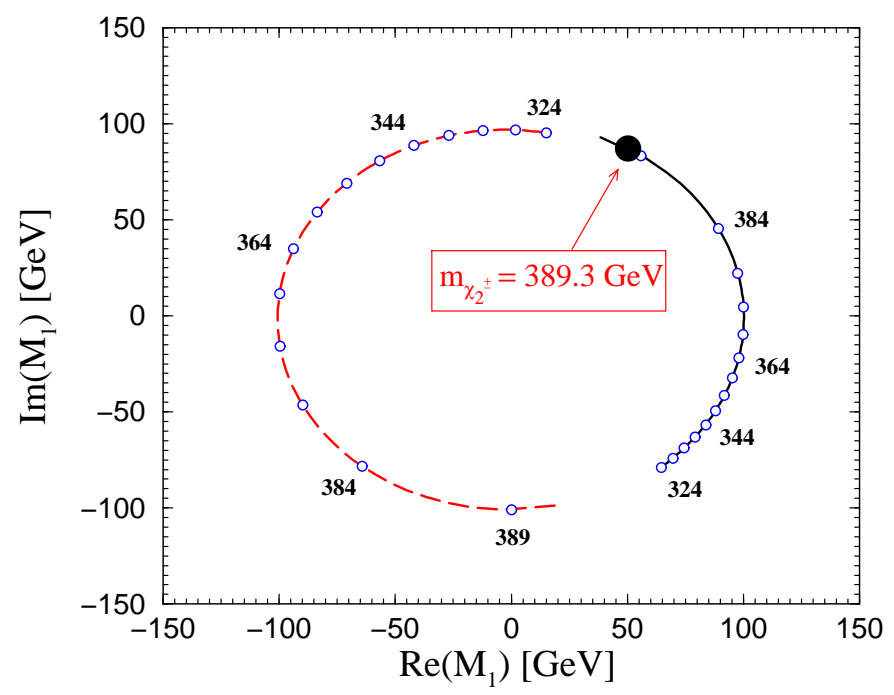

Fig. 3. The two trajectories of the crossing points of the two circles for the masses $m_{\tilde{\chi}_{1}^{0}}$ and $m_{\tilde{\chi}_{2}^{0}}$ in the $\left\{\Re \mathrm{e} M_{1}, \Im m_{1}\right\}$ plane, parameterized by the heavy chargino mass $m_{\tilde{\chi}_{2}^{ \pm}}$. The small open circles denote the heavy chargino mass parameter spaced by $5 \mathrm{GeV}$; the unique solution which is determined by the measurement of the pair-production cross sections, is marked by the black dot

(B) However, in scenarios with $C P$ violation, the loci of the two crossing points depend on the unknown heavy chargino mass $m_{\tilde{\chi}_{2}^{ \pm}}$. The two values of $\Re \mathrm{e} M_{1}$ and $\Im \mathrm{m} M_{1}$ are depicted for the window of the allowed values $m_{\tilde{\chi}_{2}^{ \pm}}$in the two upper panels Fig. 2a,b assuming that the sign of $\sin \Phi_{\mu}$ will have been determined [cf. Footnote \# 4].

By measuring the pair-production cross sections $\sigma_{L}\left\{\tilde{\chi}_{1}^{0} \tilde{\chi}_{2}^{0}\right\}$ and $\sigma_{R}\left\{\tilde{\chi}_{1}^{0} \tilde{\chi}_{2}^{0}\right\}$, a unique solution, for both the parameters $m_{\tilde{\chi}_{2}^{ \pm}}$and $\Re \mathrm{e} M_{1}, \Im \mathrm{m} M_{1}$ can be found at the same time as demonstrated in the lower panels Fig. 2c,d. The second solution $m_{\tilde{\chi}_{2}^{ \pm}} \approx 335 \mathrm{GeV}$ for $\sigma_{L}\left\{\tilde{\chi}_{1}^{0} \tilde{\chi}_{2}^{0}\right\}$ can be excluded by the measurement of $\sigma_{R}\left\{\tilde{\chi}_{1}^{0} \tilde{\chi}_{2}^{0}\right\}$ because the predicted value of $13.2 \mathrm{fb}$ is far away from its experimental value of $16.4 \mathrm{fb}$. The cross sections depend on the neutralino mixing parameters which are given by the fundamental U(1) and SU(2) gaugino and higgsino parameters. They are parametrized therefore solely by $m_{\tilde{\chi}_{2}^{ \pm}}$after the chargino system and the two neutralino masses $m_{\tilde{\chi}_{1,2}^{0}}$ are evaluated as elaborated before. As a result, the additional measurement of the cross sections leads to a unique solution for $m_{\tilde{\chi}_{2}^{ \pm}}$and subsequently to a unique solution for $\left\{M_{1}, M_{2} ; \mu ; \tan \beta\right\}$ [assuming that the discrete CP ambiguity in the associated signs of $\sin \Phi_{\mu}$ and $\sin \Phi_{1}$ has been resolved by measuring the normal $\tilde{\chi}_{2}^{0}$ polarization].

This procedure can nicely be summarized in a single figure: Figure 3 . The two crossing points of the masses $m_{\tilde{\chi}_{1}^{0}}$ and $m_{\tilde{\chi}_{2}^{0}}$ in Fig. 1 define the two trajectories in the complex $M_{1}$ plane, parametrized by the heavy chargino mass $m_{\tilde{\chi}_{2}^{ \pm}}$. The $L$ and $R$ cross sections vary along the two trajectories; comparing the predicted values with the measured values leads to a unique solution on the trajectories marked by a black dot, i.e. to unique values for $M_{1}$ and $m_{\tilde{\chi}_{2}^{ \pm}}$, along with finally unique solutions for $M_{2}, \mu$ and $\tan \beta$.

To summarize. If only the light chargino $\tilde{\chi}_{1}^{ \pm}$and the two light neutralinos $\tilde{\chi}_{1}^{0}$ and $\tilde{\chi}_{2}^{0}$ can be accessed kinematically in the initial phase of $e^{+} e^{-}$linear colliders, measurements of the masses $m_{\tilde{\chi}_{1}^{0}}$ and $m_{\tilde{\chi}_{2}^{0}}$, and the neutralino production cross section and $\tilde{\chi}_{2}^{0}$ polarization in the process $e^{+} e^{-} \rightarrow$ $\tilde{\chi}_{1}^{0} \tilde{\chi}_{2}^{0}$, combined with the light chargino mass $m_{\tilde{\chi}_{1}^{ \pm}}$and the chargino production cross section of the process $e^{+} e^{-} \rightarrow$ $\tilde{\chi}_{1}^{+} \tilde{\chi}_{1}^{-}$for polarized beams, allow us to perform a complete and precise analysis of the basic MSSM parameters in the gaugino/higgsino sector: $\left\{M_{1}, M_{2} ; \mu ; \tan \beta\right\}$.

Acknowledgements. SYC is supported by the Korea Science and Engineering Foundation (KOSEF) through the KOSEFDFG collaboration project, Project No. 20015-111-02-2, JK by the KBN Grant No. 2P03B 060 18. The work is supported in part by the European Commission 5-th Framework Contract HPRN-CT-2000-00149.

\section{References}

1. S.Y. Choi, J. Kalinowski, G. Moortgat-Pick, P.M. Zerwas, Eur. Phys. J. C 22, 563 (2001)

2. TESLA Technical Design Report, Part III: Physics at an $e^{+} e^{-}$Linear Collider, eds. R.-D. Heuer, D. Miller, F. Richard, P. Zerwas, DESY 2001-011 [hep-ph/0106315]

3. G. Moortgat-Pick, H. Steiner, EPJdirect C 6, 1 (2001) [hep-ph/0106155]; TESLA Technical Design Report, Part II: The Accelerator, eds. R. Brinkmann, K. Flöttmann, J. Rossbach, P. Schmüser, N. Walker, H. Weise, DESY 2001011 [hep-ph/0106315]

4. E. Accomando et al., Phys. Rep. 299, 1 (1998); B.C. Allanach et al., The SPS: Benchmarks for SUSY Searches, hep-ph/0202233, Eur. Phys. J. C., in press

5. G. Moortgat-Pick, A. Bartl, H. Fraas, M. Majerotto, Eur. Phys. J. C 9, 521 (1999) [Err. ibid. 549]; J.L. Kneur, G. Moultaka, Phys. Rev. D 59, 015005 (1999); D 61, 095003 (2000)

6. S.Y. Choi, A. Djouadi, M. Guchait, J. Kalinowski, H.S. Song, P.M. Zerwas, Eur. Phys. J. C 14, 535 (2000)

7. S.Y. Choi, A. Djouadi, H. Dreiner, J. Kalinowski, P.M. Zerwas, Eur. Phys. J. C 7, 123 (1999); S.Y. Choi, A. Djouadi, H.S. Song, P.M. Zerwas, Eur. Phys. J. C 8, 669 (1999); S.Y. Choi, M. Guchait, J. Kalinowski, P.M. Zerwas, Phys. Lett. B479, 235 (2000)

8. Y. Kizukuri, N. Oshimo, Phys. Lett. B249, 449 (1990); T. Ibrahim, P. Nath, Phys. Rev. D 58, 111301 (1998) [Err. ibid. 60, 099902 (1998)]; T. Ibrahim, P. Nath, Phys. Rev. D 61, 093004 (2000); M. Brhlik, G.J. Good, G.L. Kane, Phys. Rev. D 59, 115004 (1999); R. Arnowitt, B. Dutta, Y. Santoso, Phys. Rev. D 64, 113010 (2001); S. Abel, S. Khalil, O. Lebedev, Nucl. Phys. B606, 151 (2001) and references therein.

9. S.Y. Choi, H.S. Song, W.Y. Song, Phys. Rev. D 61, 075004 (2000); V.D. Barger, T. Falk, T. Han, J. Jiang, T. Li, T. Plehn, Phys. Rev. D 64, 056007 (2001)

10. G. Moortgat-Pick, A. Bartl, H. Fraas, M. Majerotto, Eur. Phys. J. C 18, 379 (2000) 